\title{
LAS FIGURAS DE CALIDAD AGROALIMENTARIA DIFERENCIADAS COMO HERRAMIENTAS PARA UN DESARROLLO RURAL SOSTENIBLE
}

\begin{abstract}
J. J. Nicasio*
Servicio de Calidad Agroalimentaria de la Dirección General de Desarrollo Rural y Política Agraria Común de la Conselleria de Agricultura, Medio Ambiente, Cambio Climático y Desarrollo Rural. C/ Castán Tobeñas, 77 Edif. B4 2º 46018 Valencia

*nicasio_jio@gva.es
\end{abstract}

\section{Resumen}

La situación actual del sector agroalimentario nos lleva a la necesidad de agudizar el ingenio para encontrar fórmulas que permitan que los consumidores puedan identificar nuestros productos en el mercado. Una fórmula fundamental para conseguir dicho objetivo es el recurso a las figuras de calidad agroalimentarias diferenciadas. Dada la pluralidad de figuras existentes en el mercado es muy importante saber identificar cuál de ellas es la que mejor se adapta a nuestro producto, y a la imagen que queremos que tengan del mismo sus destinatarios. Con dicha finalidad es importante delimitar cuales son las principales características de cada una de ellas, o al menos de las más importantes, así como identificar cuáles pueden ser los principales problemas con los que nos podemos encontrar a la hora de optar por una fórmula concreta. En cualquier caso, sea cual sea la fórmula adoptada, el éxito no siempre está garantizado, por lo que es más que recomendable ir paso a paso. Iniciar el proceso a través de la constitución de una marca colectiva o de garantía como experiencia previa a dar el salto hacia horizontes más ambiciosos como puedan ser una indicación geográfica protegida o una denominación de origen protegida.

Palabras Clave: trazabilidad, seguridad, credibilidad, certificación, organización de productores, desarrollo rural y sostenibilidad. 


\section{Abstract}

The current situation of the agri-food sector leads us to the need to sharpen the ingenuity to find tools that allow consumers to identify our products in the market. A fundamental tool for achieving this objective is the use of differentiated agri-food quality figures. Given the plurality of figures on the market, it's very important to know how to identify which of them is the one that best fits our product, and the image that we want that they have of the same one. With this purpose it is important to delimit what are the main characteristics of each of them, or at least of the most important ones, as well as to identify which can be the main problems with which we can find when opting for a concrete formula. In any case, whatever the formula adopted, success is not always guaranteed, so it is more than advisable to go step by step. Initiate the process through the constitution of a collective mark or guarantee as a previous experience to make the leap towards more ambitious horizons such as a protected geographical indication or protected designation of origin.

Keywords: traceability, safety, credibility, certification, producer organization, rural development and sustainability.

\section{1.- INTRODUCCIÓN}

Como en la mayoría de ocasiones el punto de partida a la hora de hablar de la necesidad de diferenciarse en el mercado parte de analizar la concreta situación en la que se encuentra nuestro sector agroalimentario. Hablar de la grave situación que atraviesa el mismo ya constituye una tónica habitual. Tal vez la novedad sería hablar de lo contrario y decir que ahora sí los precios obtenidos por el productor retribuyen de forma honesta $\mathrm{y}$ correcta el trabajo desarrollado. No hablamos de años sino de décadas de crisis del sector, lo que hace que debamos concluir que el principal problema radica en el propio sistema de reparto de beneficios a través de los distintos operadores intervinientes en la cadena alimentaria. De hecho es obvio, pero las diferentes medidas adoptadas hasta la fecha no han hecho sino mantener unos estándares de comportamiento en el mercado que sólo han servido para parchear determinadas cuestiones coyunturales sin entrar en muchas ocasiones en el fondo del problema. La globalización y la competencia en los mercados, la escasa rentabilidad de las producciones, lo que provoca el abandono de los cultivos y el envejecimiento progresivo de los trabajadores del campo, la necesidad de un mayor estímulo de la 
innovación y la investigación hacía variedades comercialmente más rentables, la lucha contra las plagas y las inclemencias meteorológicas, la necesaria protección medioambiental y la necesidad de avanzar hacía fórmulas más sostenibles de producción, junto a la necesidad de proteger las técnicas y cultivos tradicionales, hacen que el productor, si bien debe adoptar un comportamiento proactivo, deba contar con el apoyo de las distintas instituciones públicas, así como de los diferentes instrumentos articulados por éstas.

\section{2.- MECANISMOS DE REACCIÓN}

Ante este panorama tan desolador podríamos hablar de la existencia de diferentes sistemas de reacción, aunque podemos resumirlos fundamentalmente en dos:

a) La concentración de la oferta mediante fórmulas asociativas de productores agrarios (cooperativas, asociaciones de productores, organizaciones de productores de frutas y hortalizas...) cuya finalidad última no es otra que aunar esfuerzos y responder de forma conjunta a las necesidades del mercado. Todo ello sin olvidar el importante papel que juegan a la hora de reducir costes, tanto en efectivos humanos como materiales, y de aumentar su especialización, planificación y orientación al mercado ${ }^{1}$.

b) La diferenciación con los competidores. Hay múltiples formas de diferenciarse en el mercado pero sin duda las dos más importantes son la diferenciación por precios y la diferenciación por calidad. Descartando evidentemente la diferenciación por precios, ya que nada tenemos que hacer con nuestros competidores de "allende los mares", debemos diferenciar nuestros productos en base a su calidad. Pero qué significa diferenciarse por calidad? Podríamos buscar múltiples definiciones más o menos académicas de lo que se entiende por calidad, pero tal vez lo más sensato sería preguntar a los propios consumidores que es lo que entienden como tal, ya que son ellos quienes finalmente van a adquirir nuestros productos en función de la misma. Así pues, partiendo de esta idea podríamos entender la calidad como el conjunto de atributos de valor y satisfacción que diferencian a los productos en función de múltiples factores, algunos socio-culturales (alimentos elaborados o producidos a través de métodos tradicionales y vinculados al ámbito rural), medioambientales (elaborados siguiendo

De ello sabe mucho la Cooperativa Valenciana Unió Protectora d'El Perelló (UNIPRO). 
sistemas sostenibles y respetuosos con el medio ambiente y la protección de la flora y la fauna), éticos (en función de las condiciones de trabajo y de comercio justos), o energéticos (compatibles con una cultura basada en el deporte y la vida saludable).

Ahora bien, si bien el concepto puede ser todo lo amplio y diferente que podamos imaginar, toda definición lleva implícito un elemento fundamental, la seguridad. Y es que los consumidores están cada vez más preocupados porque los productos que consumen posean unas garantías mínimas de inocuidad y de que los mismos no constituyan una amenaza para la salud. De ahí la importancia que juega la palabra "trazabilidad" en todo alimento, trazabilidad que no es otra cosa que la capacidad de seguir el proceso completo de un alimento, a través de todas las etapas de producción, transformación, envasado y comercialización, lo que incluye también su almacenamiento, transporte, venta y cualquier otro tipo de entrega a título oneroso o gratuito de dicho producto. Y esta capacidad debe hacerse extensiva a sus ingredientes, materias primas, aditivos y otras sustancias destinadas a formar parte del mismo.

Así pues, producto local, tradicional, obtenido de forma sostenible y respetuosa con el medio ambiente sí, pero seguro y debidamente trazado, para lo cual necesitamos que el mismo esté amparado por un sello de calidad que garantice la realidad de todos aquellos extremos que dice atesorar.

\section{3.- PRINCIPALES SISTEMAS DE CALIDAD}

Pero llegados a este punto, y una vez sabido que debemos diferenciar nuestros productos por su calidad ¿qué sello de calidad debemos elegir?. En el mercado existe un número ingente de sellos y figuras de calidad, km. 0, slow food, producto de proximidad, artesano, marcas colectivas, marcas de garantía, denominaciones de origen protegidas, indicaciones geográficas protegidas, agricultura ecológica, comercio justo, especialidades tradicionales garantizadas, productos de montaña, productos de granja, y un sinfín de sellos, pero al final el que elegiremos será aquél que resulte de la combinación de múltiples factores entre los que podemos destacar los atributos que queremos que trasciendan de nuestro producto al consumidor, las exigencias de nuestros compradores y distribuidores, y del mercado y, evidentemente, el coste que nos va a reportar dicho sello y si el mismo compensa el esfuerzo dedicado a su obtención. 
Con objeto de relacionar los más importantes y sus principales características, y sin pretender hacer una relación exhaustiva de los mismos, vamos a referirnos brevemente a algunos de los marchamos de calidad con los que nos podemos encontrar en el mercado haciendo, una primera diferenciación entre sellos de calidad de carácter privado y sellos de calidad de carácter público.

\section{1.- Sellos de carácter privado}

a) En primer lugar tenemos los sistemas individuales de certificación que garantizan que una determinada empresa o marca cumple unos determinados estándares de seguridad y trazabilidad exigidos por sus clientes para su puesta en el mercado. Algunos de los más conocidos son el Estándar Mundial BRC para la seguridad de los alimentos o la norma IFS (International Food Standard). Se trata de normas comunes de calidad y seguridad alimentaria que ayudan a las compañías a seleccionar y calificar a sus productores.

Si bien estos son los más conocidos hay muchos otros que certifican aspectos concretos de la actividad productiva como por ejemplo certificaciones de producciones locales (Km.0, slow food, venta local, proximidad...), elaborados de forma artesana o tradicional, o vinculadas al comercio justo. En estos casos tan importantes es la marca que usa estas certificaciones como la propia entidad certificadora y la seguridad que la misma aporta al consumidor. De nada sirve un signo identificativo de que un producto cumple una determinada especificación, si el mismo no es conocido y la entidad que lo certifica no nos dice nada. De ahí la importancia que tiene su promoción a nivel global.

b) De carácter colectivo, destacando las Marcas Colectivas y las Marcas de Garantía privadas recogidas en el Título VII (artículos 62 a 78) de la Ley 17/2001, de 7 de diciembre, de Marcas.

Tanto unas como otras son adoptadas por una colectividad o agrupación de productores para la gestión de un determinado producto o gama de productos, los cuales poseen un origen $\mathrm{u}$ otra característica particular común a todos ellos. Realmente, la constitución de estas marcas constituye en la práctica una excelente experiencia previa para comenzar a trabajar de forma conjunta y constituir un primer paso para alcanzar otras metas más ambiciosas como puedan ser la creación de una marca de garantía pública o de una indicación protegida (denominación de origen protegida o indicación geográfica protegida). La principal herramienta de 
estas marcas es su reglamento de uso. Se trata del conjunto de disposiciones o normas internas que pretenden unificar o estandarizar el uso de la marca, por parte de los asociados, con relación a los productos de que se trate, partiendo de los elementos de calidad, homogeneidad, estabilidad u otras características particulares que se pretenda que la marca englobe o anuncie al consumidor o a sus potenciales clientes. Así pues, forman parte de su contenido, entre otros aspectos, las condiciones y modalidades de uso de la marca, las condiciones de afiliación, las personas autorizadas a su uso, los mecanismos para garantizar y controlar sus uso adecuado, consecuencias del incumplimiento de lo dispuesto en el mismo, cuotas, etc. Y lo importante del mismo es que todo este contenido es determinado de forma voluntaria por la propia organización y/o asociación de productores. Ahora bien, en el caso de las marcas de garantía su reglamento de uso debe ser informado favorablemente por el órgano administrativo competente en atención a la naturaleza de los productos o servicios a los que la marca de garantía se refiere. En este caso por la Consellería competente en materia de agricultura.

Como ejemplos de marcas colectivas podemos citar la Alcachofa Vega Baja del Segura, el Pimiento de Torquemada, la Patata de Burgos, o el tomate de los Palacios y el tomate rosa de Barbastro. Y como marcas de garantía Tomazur, el Aceite de Huelva, el Cochinillo de Segovia, el Chorizo Zamorano o las Setas de Castilla León, aunque su número también es ingente.

\section{2.- Sellos de carácter público}

a) Marcas Colectivas y de Garantía Públicas:

Las distintas Comunidades Autónomas tratan de promover la calidad de los alimentos producidos en ellas no sólo por su importancia a nivel económico sino también por su papel como instrumento de fijación de la población en el territorio. En definitiva intentan contribuir al desarrollo económico de sus zonas rurales incentivando el uso de los diferentes sellos de calidad y origen existentes, con el fin de mantener o incluso incrementar la producción agroalimentaria de calidad diferenciada y contribuir a su promoción en los mercados tanto locales como nacionales e internacionales, así como preservar y revalorizar su contribución al patrimonio y tradición autóctonos. 
Con esta finalidad, el 26 de junio de 1998, se publicó el el Diari Oficial de la Generalitat Valenciana el Decreto 91/1998, de 16 de junio, del Gobierno Valenciano, por el que se aprobaba el Reglamento de la Marca de Calidad "CV" para productos agrarios y agroalimentarios, Decreto que sigue actualmente en vigor aunque debe ser objeto de una profunda revisión con el fin de adaptarlo a la nueva normativa y realidades del sector.

$\mathrm{Su}$ finalidad es, tal y como reza el preámbulo de dicha norma, ofrecer a los productores, elaboradores y transformadores de productos agrarios y alimentarios, la posibilidad de diferenciar su oferta comercial, ofreciendo al consumo, con criterios de calidad, productos con una especial identificación cualitativa garantizada.

Entre sus principales características podemos citar las siguientes:

- Titularidad pública de las mismas, en concreto de la Conselleria con competencias en materia de agricultura.

- Necesaria aprobación previa de una reglamentación técnica de calidad que establezca las características que debe cumplir el producto amparado.

- Contratación de una entidad de carácter independiente e imparcial, con capacidad técnica y económica acreditadas, que realice el control externo del cumplimiento del régimen de la marca de calidad, y en especial de la reglamentación técnica previamente aprobada.

- Creación y/o constitución de una asociación de productores ("entidad asociativa gestora de la autorización del uso de la marca CV") que se encargará de desarrollar las actividades de promoción comercial del producto o productos amparados, y de velar por el correcto uso de la marca de calidad por sus asociados, adoptando para ello las medidas de control establecidas en sus correspondientes estatutos.

Sin duda alguna los elementos que hacen a un grupo de productores optar por esta fórmula asociativa en lugar de recurrir a otras fórmulas de carácter privado es, sin duda, el hecho de que las mismas suponen para los consumidores una mayor garantía de credibilidad sobre la naturaleza, alcance y seguridad de los productos amparados (medio institucionalizado de reconocimiento e identificación de los productos de calidad), sin olvidar los importantes beneficios de carácter público que también pueden derivarse

Por lo general estaremos hablando de la misma asociación promotora de la creación de la marca CV. 
de ella, como por ejemplo el acceso a diferentes lineas de ayudas públicas. Por contra, y de forma lógica, la libertad de la asociación gestora de la marca a la hora de regular y delimitar los distintos aspectos que la integran queda limitada por la labor supervisora de la propia administración, así como por la labor ejercida por la entidad independiente encargada de realizar su control externo ${ }^{3}$.

Como Marcas CV de la Comunitat Valencian podemos citar, a título de ejemplo, la tomata de penjar d'Alcalà de Xivert, el Coent de Burriana, la Asociación Gastronómica de Productos de Pinoso, la Carne de Conejo CV o la Clóxina de Valenciana.

Hemos hablado de la Marca CV para productos agroalimentarios de la Conselleria con competencias en materia de agricultura pero hay otros departamentos del Consell que han creado otras figuras con funciones y papeles muy similares, tal sea el caso de la Marca Paques Naturales para productos elaborados en el ámbito de los parques naturales de la Comunitat Valenciana o su área de influencia ${ }^{4}$, o la artesanía agroalimentaria a través de la obtención del Documento de Calificación Artesana (D.C.A.).

b) Figuras de calidad Comunitarias:

Especial mención merecen los distintos signos que hacen referencia a las indicaciones geográficas reconocidas por la normativa comunitaria, y ello por su importancia en el tráfico económico y de reputación e información. Estas figuras de calidad presentan un innegable valor promocional por su grado de caracterización tanto al referirse al lugar de origen, cultivo, elaboración o fabricación del producto agroalimentario, como a su cualificación, aspectos que hacen que puedan ser fácilmente diferenciables respecto de otros productos del mismo género pero procedentes de otras zonas geográficas.

Sin duda alguna las figuras más conocidas son las denominaciones de origen protegidas (DOP's) y las indicaciones geográficas protegidas (IGP's), siendo la principal diferencia entre ambas, tal y como establece el Reglamento (UE) $\mathrm{n}^{\mathrm{o}}$ 1151/2012, sobre los regímenes de calidad de los

\footnotetext{
${ }^{3}$ Esta labor de control externo es la que provoca, en ocasiones, reticencias importantes a la hora de constituir una marca CV, ya que comporta la necesidad de sufragar los costes que genera esta actuación. No obstante lo anterior, también existen distintas lineas de ayudas públicas encargadas precisamente de sufragar, sino todos, parte de los gastos generados por las tareas de control y certificación soportadas.

${ }^{4}$ Marca que pueden utilizar los tomates del Perelló en función de su enclave dentro del Parque Natural de l'Albufera o de su área de influencia, y de su privilegiado microclima.
} 
productos agrarios y alimentarios, el hecho de que mientras la denominación de origen protegida es un nombre que identifica un producto originario de un lugar determinado, una región o, excepcionalmente un país, cuya calidad o características se deben fundamentalmente o exclusivamente a un medio geográfico particular, con los factores naturales y humanos inherentes a él, y cuyas fases de producción tienen lugar en su totalidad en la zona geográfica definida, en el caso de la indicación geográfica protegida es suficiente que posea una calidad determinada, una reputación u otra característica que puedan esencialmente atribuirse a su origen geográfico, y de cuyas fases de producción, una al menos tenga lugar en la zona geográfica definida. Ahora bien, no significa con esto que una denominación de origen protegida represente un nivel superior de protección que una indicación geográfica protegida, ya que en muchas ocasiones hacen referencia y amparan realidades diferentes, sino simplemente que en el caso de una denominación de origen protegida el vínculo entre el producto y el ámbito geográfico es más fuerte o estricto. Imaginemos el caso de dos figuras de calidad existentes en la Comunitat Valenciana como puedan ser el Turrón de Alicante y Jijona y el Kaki de la Ribera del Xúquer. En el primer caso estamos hablando de dos Indicaciones Geográficas Protegidas que identifican un producto transformado (turrón) elaborado en el municipio de Jijona de una forma muy concreta y tradicional, en el que juegan un papel fundamental la reputación y saber hacer de sus gentes (factores humanos inherentes al mismo). Pero para su elaboración hacen falta determinadas materias primas, como puedan ser la miel o la almendra, que pueden proceder de otras áreas geográficas diferentes. Por tanto, las condiciones edafo-climáticas propias del municipio de Jijona no juegan un papel determinante a la hora de obtener dicho producto. No es el caso de la denominación de origen protegida Kaki de la Ribera del Xúquer, en el que al tratarse de un producto fresco cultivado en un área geográfica muy concreta y determinada debe sus características particulares a las concretas condiciones edafo-climáticas existentes en los municipios y territorios que forman parte de la misma. Así pues, en muchas ocasiones, el tipo de figura más adecuada dependerá del tipo de producto y de las necesidades concretas del sector.

Pero aunque las denominaciones de origen protegidas e indicaciones geográficas protegidas sean las más conocidas, existen otras figuras de calidad que es conveniente conocer. Tal es el caso de las Especialidades Tradicionales Garantizadas (ETG's) o la Agricultura de Montaña. 
Tal vez el término ETG sea el menos conocido, sin embargo a todos nos suena el término "Jamón Serrano". Pues bien, cuando hablamos de jamón serrano estamos hablando de otra figura de calidad amparada por la normativa comunitaria, las especialidades tradicionales garantizadas, que tal y como establece el Reglamento (CE) 1151/2012 ya citado, son aquellos productos resultado de un método de producción, transformación $\mathrm{o}$ composición que correspondan a la práctica tradicional aplicable a ese producto o alimento, o estén producidos con materias primas o ingredientes que sean utilizados tradicionalmente. Así pues, para que se admita el registro como ETG de un nombre, este deberá haberse utilizado tradicionalmente para referirse al producto específico, o identificar el carácter tradicional o específico del mismo. Aparte del Jamón Serrano, no son muchas las ETG's existentes en nuestro país pero también podemos citar la leche de granja, els panellets o las tortas de aceite de Castilleja de la Cuesta.

Por último, el citado Reglamento (UE) $n^{\circ} 1151 / 2012$ establece un régimen de términos de calidad facultativos para facilitar la comunicación por sus productores, dentro del mercado interior, de las características o atributos de los productos agrícolas que aporten valor añadido fijando condiciones de utilización del término de calidad facultativo «producto de montaña», norma que ha sido completada a través del Reglamento Delegado (UE) $n^{0} 665 / 2014$, de la Comisión de 11 de marzo en lo que atañe a las condiciones de utilización de dicho término, si bien se deja en manos de los distintos Estados Miembros el establecimiento de la normativa concreta para su puesta en práctica.

Pero una vez llegados a este punto, ¿qué es lo que da a estas figuras de calidad tanta notoriedad y las lleva a posicionarse en la cúspide de las figuras de calidad agroalimentarias diferenciadas? Sin duda aquí vuelve a jugar un papel fundamental el control que sobre las mismas realizan las instancias públicas (garantía institucional), y en este caso no sólo las autoridades autonómicas sino también las nacionales y, sobre todo, las comunitarias. Hablamos en este caso de un sistema de control y certificación mucho más estricto y que es fácilmente identificable por el mercado, lo que facilita las ventas. De hecho, y a pesar de que como cualquier otro sistema no está exento de crítica, lo cierto es que anualmente más del $10 \%$ de los productos que podemos encontrar a la venta utilizando los logos comunitarios son falsificaciones, lo que demuestra el interés que por los mismos tiene el mercado. 
Otra de sus principales características es su sistema de organización. En el caso de la Comunitat Valenciana, el mismo viene establecido en el Decreto 222/2007, de 9 de noviembre, del Consell, por el que se establecen normas relativas a los Consejos Reguladores u Órganos de Gestión de las denominaciones de calidad de la Comunitat Valenciana, en su redacción dada por el Decreto 46/2010, de 12 de marzo. Esta norma configura a los Consejos Reguladores de las DOP's e IGP's como corporaciones de derecho público, con personalidad jurídica propia y plena capacidad para el desarrollo de sus fines, pudiendo realizar toda clase de actos de gestión y administración, sujetando su actividad fundamentalmente al derecho privado, salvo aquellas funciones que tengan encomendadas que impliquen el ejercicio de potestades o funciones públicas, tal es el caso de las funciones de certificación o de llevanza de los registros de sus operadores, en cuyo caso se someten al derecho público.

Los Consejos Reguladores u Órganos de Gestión de nuestras figuras de calidad diferenciada agroalimentaria reconocidas (DOP's, IGP's y Comité de Agricultura Ecológica de la Comunitat Valenciana), deben tener una estructura y funcionamiento democráticos, rigiendo en todo momento los principios de representación paritaria de todos los sectores implicados. La organización mínima de cada Consejo Regulador u órgano de gestión está constituida por el Pleno, el/la Presidente/a, el/la Vicepresidente/a, el/la Secretario/a del Pleno, y dos vocales técnicos designados por la conselleria competente en materia de agricultura y alimentación, con voz pero sin voto, los cuales velan por el cumplimiento de la legislación y normativa aplicables, y además, pueden contar con un gerente y cualquier otro órgano que se establezca en sus Estatutos.

Pero al igual que ocurría con el resto de figuras de calidad, una de las tareas fundamentales, por no decir la más importante y la que constituye la esencia de cualquier indicación geográfica son las tareas de control y certificación, que dan fe de que el producto amparado en cuestión cumple estrictamente con todos los requisitos exigidos en su normativa técnica (pliego de condiciones técnicas). Esta es la norma que define sus características diferenciales, su ámbito geográfico delimitado y establece el vínculo existente entre los diferentes elementos que conforman su contenido y razón de ser. Así pues, el consejo regulador u órgano de gestión está obligado a establecer el correspondiente sistema de control y certificación, que, en todo caso, estará separado del sistema de gestión del mismo. Dicho control y certificación puede ser ejercido: 
a) Por un organismo público, que actuará de acuerdo con los principios establecidos en la normativa reguladora del control oficial de los productos alimenticios.

b) Por un órgano de control integrado en el consejo regulador $u$ órgano de gestión, que deberá encontrarse adecuadamente separado del citado órgano de gestión con objeto de garantizar su independencia. De hecho sus tareas se realizarán sin ninguna dependencia jerárquica ni administrativa respecto de los órganos de dirección del consejo regulador y siempre bajo la tutela de la administración competente. En este caso, dicho órgano de control deberá acreditarse en la norma UNE-EN ISO/IEC 17065:2012 (Evaluación de la conformidad. Requisitos para organismos que certifican productos, procesos y servicios. (ISO/IEC 17065:2012).

c) Por un organismo independiente de control inscrito como tal en el registro correspondiente.

d) Por un organismo independiente de inspección, acreditado en el cumplimiento de la norma sobre criterios para el funcionamiento de los diversos tipos de organismos que realizan inspección (UNE-EN ISO/IEC 17020 o norma que la sustituya).

\section{4.- CONTRIBUCIÓN AL DESARROLLO RURAL.}

Una vez relacionadas brevemente algunas de las principales figuras de calidad agroalimentarias diferenciadas podemos recuperar el argumento inicial referente a la existencia de dos formas fundamentales de reacción contra la situación en la que nos encontramos, la concentración de la oferta y la diferenciación basada en la calidad Pues bien, tanto una asociación de productores integrados en una marca colectiva o de garantía, ya sea pública o privada, como un Consejo regulador o un órgano de gestión de una denominación de origen protegida o de una indicación geográfica protegida son ambas cosas. Son un instrumento de concentración de la oferta, mediante la creación de una agrupación de productores en la que, en no pocas ocasiones, forman parte los operadores de otros ámbitos de la cadena alimentaria (comercializadores, envasadores, distribuidores), lo que da más fuerza a la organización, y son un instrumento de diferenciación basado en la calidad de los productos ofrecidos al consumidor final. Y además, $\underline{\text { son }}$ una herramienta para el desarrollo rural sostenible, y ello fundamentalmente por los siguientes motivos: 
a) Porque son el resultado de un proceso de identificación territorial con la creación de marcas con las que el territorio se promociona de forma directa e indirecta.

b) Porque los métodos locales utilizados para producirlos crean un vínculo de confianza entre el consumidor, el productor, el lugar de donde es originario, y las personas que allí viven y lo producen.

c) Porque garantizan productos con unas específicas características de producción, procesamiento, preparación y presentación, y vinculados a su vez con un concreto ámbito geográfico.

d) Reportan claros beneficios medioambientales al tratarse de productos de proximidad (huella de carbono, kilómetro 0, prácticas de cultivo compatibles con el medio ambiente, etc.).

e) Y ejercen una función de interlocutores válidos con las distintas instituciones públicas, participando en las distintas estrategias que se desarrollan sobre el territorio y ámbito rural.

Estas funciones unidas a la necesidad de concentración sectorial hacen que las figuras de calidad agroalimentaria diferenciadas de carácter público sean canalizadoras de múltiples ventajas y ayudas públicas dirigidas precisamente a hacer más visible su labor.

Todos estos elementos pueden ser identificados en un producto como es el de la "tomata del perelló". Un producto diferente (carnoso, con poca semilla, piel fina y sabor dulce con poca acidez), fruto de una elaboración y cuidado tradicional en el que el minifundio constituye una pieza fundamental, ubicado en un enclave privilegiado $\mathrm{y}$ con unas condiciones edafo-climáticas que no se dan en otros lugares (tierras dunares, aguas salitrosas,...), y obtenido mediante la utilización de técnicas respetuosas con el medio ambiente ${ }^{5}$ y su entorno ("Parc Natural de l'Albufera"). Todo ello sin olvidar su evidente componente social y cultural, vinculado con las fiestas, tradiciones y costumbres locales, y que llegan a constituir señas de identidad y de identificación de sus habitantes con su medio.

Por ejemplo mediante la lucha biológica contra plagas como la tuta. 


\section{5.- CONCLUSIÓN}

Pero, llegados a este punto, ¿cuál debe ser el camino que debe seguir un determinado producto para acceder a los mercados y consolidarse en los mismos? ¿Es el recurso a la figura de calidad garantía de éxito?

Como todo en la vida no hay recetas milagrosas ni soluciones únicas que garanticen el éxito. No son pocas las figuras de calidad agroalimentarias a nivel europeo, por ejemplo denominaciones de origen protegidas, que una vez creadas y registradas por la Comisión Europea han desaparecido ante la falta de apoyo de los propios operadores que la constituyeron, o porque no han sabido llegar al consumidor o al mercado. No hay que olvidar que la supervivencia de cualquier figura de calidad pasa esencialmente por el apoyo de los propios productores a la misma, tanto desde el punto de vista humano y material como económico, todo ello con independencia de que puedan contar con algún estímulo por parte de las distintas administraciones públicas. Por otro lado también hay que tener en cuenta que el proceso a seguir a la hora de alcanzar el reconocimiento por parte de la Comisión como denominaciones de origen protegidas o indicaciones geográficas protegidas es largo y costoso, y en ocasiones puede alargarse durante muchos años. Por eso es aconsejable, en la mayoría de ocasiones, comenzar a trabajar como marcas colectivas o de garantía privadas para más adelante, y previo paso por una marca de garantía pública como pueda ser la Marca $\mathrm{CV}$ de la Comunitat Valenciana ${ }^{6}$, avanzar en la consecución de una denominación de origen protegida o de una indicación geográfica protegida.

También hay que tener en cuenta que no siempre el recurso a una figura de calidad agroalimentaria diferenciada es la mejor herramienta para alcanzar los mercados (y para alcanzar al consumidor). De hecho si nos preguntaran si conocemos alguna denominación de origen de melones, kiwis, fresones o quesos, tal vez pueda ser que no nos venga ninguna a la cabeza. Sin embargo si nos hablan de marcas de productos como El Abuelo, Bollo, Zespri, Fresón de Palos, García Vaquero o Gran Capitán, tal vez nos suenen más y automáticamente identifiquemos de que producto estamos hablando. Lo que sí es cierto es que el recurso a las figuras de calidad agroalimentaria diferenciadas supone un instrumento muy útil al alcance de los pequeños productores que por sí mismos sería muy difícil que pudieran

\footnotetext{
6 Como recomendación, ya que al ser su nivel de exigencia y controles, por lo general, mayores que los de una marca colectiva o de garantía privadas, constituye una buena experiencia antes de dar el salto a las DOP's e IGP's. Evidentemente no es necesario haber sido antes Marca CV para conseguir una denominación de origen protegida.
} 
conseguir diferenciarse en el mercado, a la vez que, y tal y como se ha dicho anteriormente, son una herramienta fundamental para el desarrollo rural sostenible.

\section{REFERENCIAS BIBLIOGRÁFICAS}

Cambra, J; Villa, A. 2009. Denominaciones de origen e indicaciones geográficas: Justificación de su empleo y valoración de su situación actual en España. Caja Rural Intermediterránea, Cajamar.

Muñiz, E. 2008. Estudios Jurídicos del Derecho Agrario. En: Carretero, A. Normas de protección de los signos distintivos de calidad de los productos agroalimentarios en el derecho español. Ministerio de Medio Ambiente y Medio Rural y Marino, pp. 387-412.

Sánchez, A. 2014. Productos Agroalimentarios de calidad como mecanismos de protección de productores y de consumidores. Revista internacional de doctrina y jurisprudencia. Universidad de Almería. 